\title{
Termites reigned by royals close ranks
}

\section{Graham J. Thompson ${ }^{1}$}

Published online: 6 February 2021

(c) International Union for the Study of Social Insects (IUSSI) 2021
For many animals, recognizing kin is an important aspect of social life. So fundamental is the notion that parents should direct care towards their own offspring that we rarely stop to question this biological rule, except when it is conspicuously broken by brood parasites. Choosing a mate from outside the family is likewise common enough that rare cases of close inbreeding are curious deviations from a norm. Recognizing relatives-for affiliation or avoidance-is a mechanism that mediates many common behaviors and underpins much behavioural ecology. For eusocial insects, recognizing kin takes on added significance because the reproductively altruistic castes can only gain indirect fitness if their altruism is ultimately directed towards reproducing relatives.

Among eusocial insects, termites provide an interesting microcosm for studying how indirect selection can promote kin-recognition rules. Most species of termite have a non-reproductive worker and soldier caste, the latter being particularly specialized towards altruistic defence of their colony against intrusion by foreign foes. We can, therefore, expect these castes to have an acute sense of recognitiontheir indirect fitness depends on it. Yet, for some reason certain species of termite can be highly temperamental, at least in arena assays, to even crudely distinguish nestmates from unrelated intruders (Simkovic et al. 2018). This seemingly meek behavior suggests that many kin or nestmate recognition assays are unrealistic or that recognition is not always visible, important or possible.

By studying aggression in groups of Reticulitermes speratus, a common subterranean termite (Rhinotermitidae; Fig. 1) in Japan, Konishi and Matsuura (2021) identify one factor that seems to promote nestmate recognition. The authors conducted an experiment in which they introduced an intruder termite (a non-nestmate conspecific, a nestmate, or a different termite species) to groups of workers and soldiers in which the reproductive king and queens were either

Graham J. Thompson

graham.thompson@uwo.ca

1 Western University, London, Canada present or absent. They found that the group response to non-nestmates was heightened in the presence of royals, and that soldiers were more aggressive in this response than workers. Royals, it seems, are an important provocateur of the termite recognition and defense response, at least towards non-nestmate conspecifics. The authors note that soldiers and workers did remain uniformly aggressive towards invaders from a different species (Coptotermes formosanus), with royals present or not.

If altruistic defense is ultimately driven by indirect fitness returns, then the royal-dependent response makes sense. Roughly speaking - no royals, no reproductive returns, no recognition. The precise mechanism remains unknown but perhaps it is royal pheromones that trigger the defence response, or at least lower the threshold for its expression, particularly in soldiers. Understandably, it may be difficult for in-lab recognition assays to always consider royals in experimental treatments, but the 'semi-natural' apparatus of Konishi and Matsuura enables this and other salient qualities of a natural nest.

Going forward, we might further test the presence of royalty as a factor in studies of termite aggression towards

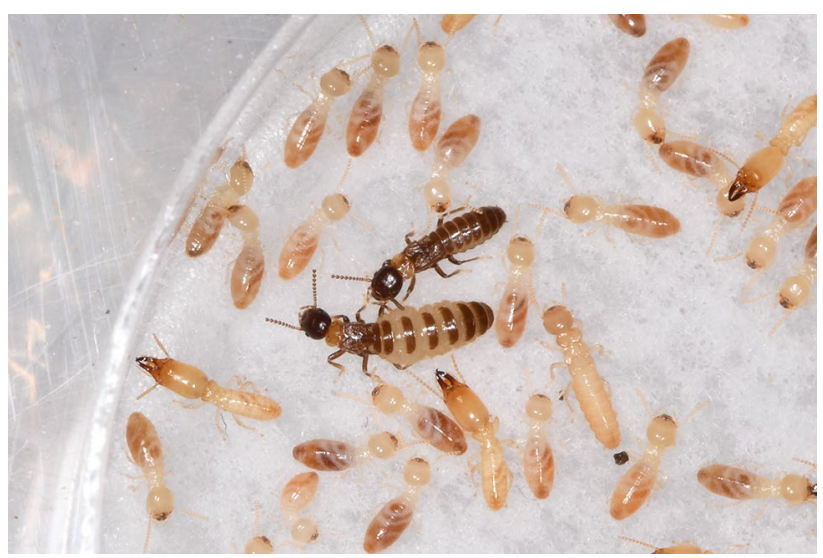

Fig. 1 Reticulitermes speratus showing a reproductive male and female (center) together with a collection of mostly workers and soldiers. Photo supplied courtesy of Takao Konishi 
non-nestmates. The effect may differ in interesting ways among castes and among species. We might also ask why royal pheromones do not appear to modulate the response against heterospecific intruders. Are different recognition rules at play? Finally, we could re-interpret past results, especially from assays involving species of Reticulitermes with 'open' societies (Clément 1986) — doing so in light of the often missing royal variable may help to clarify the disparities of past findings.

\section{References}

Clément J-L (1986) Open and closed societies in Reticulitermes termites (Isoptera, Rhinotermitidae): geographic and seasonal variations. Sociobiology 11:311-323

Konishi T, Matsuura K (2021) Royal presence promotes worker and soldier aggression against non-nestmates in termites. Insect Soc. https://doi.org/10.1007/s00040-020-00799-w

Simkovic V, Thompson GJ, McNeil JN (2018) Testing for aggression and nestmate recognition in the Eastern subterranean termite (Reticulitermes flavipes). Insect Soc 65:281-288 